\title{
含腙结构单元的氨基嘧啶衍生物的合成与生物活性研究
}

\author{
何海峰 ${ }^{b}$ 夏 芹 ${ }^{a}$ 贺红武*, $a$ \\ ( ${ }^{a}$ 华中师范大学化学学院 农药与化学生物学教育部重点实验室 武汉 430079) \\ ( ${ }^{b}$ 江西科技师范大学化学化工学院 南昌 330046)
}

\begin{abstract}
摘要 丙酮酸脱羧酶是连接糖酵解与三羧酸循环的关键酶, 目前尚无以丙酮酸脱羧酶(PDHc-E1)为靶标的杀菌剂. 拟 通过设计针对微生物 PDHc-E1 的抑制剂来获得具有杀菌活性的化合物. 以课题组前期发现的 E. coli PDHc-E1 抑制剂 L 为先导化合物进行结构修饰, 通过肼与醛的缩合反应合成了 14 个新型含腙结构单元的氨基嘧啶衍生物 I 作为潜在的 PDHc-E1 抑制剂. 发现 2-甲基-4-氨基-5-(甲基-4-溴苯腙)-嘧啶(I-6)不仅对 E. coli PDHc-E1 显示较好的活性( $\mathrm{IC}_{50}=26.45$ $\mu \mathrm{mol} / \mathrm{L})$, 同时对真菌花生褐斑 $\left(\mathrm{EC}_{50} 14.11 \mu \mathrm{g} / \mathrm{mL}\right)$ 和苹果轮纹 $\left(\mathrm{EC}_{50} 0.64 \mu \mathrm{g} / \mathrm{mL}\right)$ 显示了高效活性, 具有进一步研究的价 值. 由此, 通过对先导结构 2-甲基-4-氨基嘧啶衍生物 $\mathbf{L}$ 中的桥键进行结构修饰, 获得了对 E. coli PDHc-E1 具有抑制作 用的高效杀菌活性的化合物.
\end{abstract}

关键词 丙酮酸脱羧酶; 杀菌剂; 先导化合物; 2-甲基-4-氨基嘧啶衍生物

\section{Synthesis and Biological Evaluation of Pyrimidine Derivatives Containing Hydrazine Structural Unit}

\author{
He, Haifeng ${ }^{b} \quad$ Xia, Qin ${ }^{a} \quad$ He, Hongwu ${ }^{*, a}$ \\ ( ${ }^{a}$ Key Laboratory of Pesticide and Chemical Biology of Ministry of Education, College of Chemistry, \\ Central China Normal University, Wuhan 430079) \\ $\left({ }^{b}\right.$ School of Chemistry and Chemical Engineering, Jiangxi Science and Technology Normal University, Nanchang 330013)
}

\begin{abstract}
Pyruvate dehydrogenase multienzyme complex (PDHc) is a key enzyme linking glycolysis and the tricarboxylic acid cycle. Currently, there is no fungicide targeting pyruvate decarboxylase (PDHc-E1), the purpose of this study was to obtain compounds with bactericidal activity by designing inhibitors against PDHc-E1. On the basis of lead compound $\mathbf{L}$, fourteen novel aminopyrimidine derivatives I were designed and synthesized by the condensation reaction of hydrazine and aldehydes as potential PDHc-E1 inhibitors. The most effective 5-((2-(4-bromophenyl)hydrazono)methyl)-2-methylpyridin- 4-amine (I-6) with good $E$. coli PDHc-E1 enzyme inhibitory activity $\left(\mathrm{IC}_{50}=26.45 \mu \mathrm{mol} / \mathrm{L}\right)$ exhibited most powerful inhibitory potency against Cercospora arachidicola Hori $\left(\mathrm{EC}_{50} 14.11 \mu \mathrm{g} / \mathrm{mL}\right)$ and Physalospora piricola Nose $\left(\mathrm{EC}_{50} 0.64 \mu \mathrm{g} / \mathrm{mL}\right)$. Therefore it could obtain the compound with antifungal activity against microorganism PDHc-E1 enzyme by modifying the bridged linkage in lead structure 2-methyl-4-amino-pyrimidine derivatives $\mathbf{L}$.
\end{abstract}

Keywords PDHc-E1; fungicide; lead compound; 2-methyl-4-amino-pyrimidine derivatives

杀菌剂的应用和杀虫剂及除草剂一样，已在全球范 围内成为保护农作物丰收不可缺少的手段之一，在世界 范围内杀菌剂新品种虽层出不穷, 然而, 植物病菌是极 易产生抗性的一类微生物, 因此, 为了防止杀菌剂的抗 性发展，研发作用机理独特、广谱高效的杀菌剂己成为 国际上研究开发的重点. 在目前人们正在努力探索的杀 菌剂靶标酶中，丙酮酸脱氢酶复合物(pyruvate dehydrogenase complex, PDHc) 是一个令人关注的作用靶标. $\mathrm{PDHc}$ 是连接糖䣲解与三羧酸循环的关键酶, 也是生物 体内能量代谢过程的关键酶 ${ }^{[1,2]}$ (图 1). PDHc 主要是由三 种酶高度组合而成，其中包括丙酩酸脱羧酶 $\mathrm{E} 1$ 组分 ${ }^{[3 \sim 6]}$ (pyruvate dehydrogenase complex E1 component, PDHc-

* Corresponding author. E-mail: he1208@mail.ccnu.edu.cn

Received March 27, 2019; revised May 12, 2019; published online June 6, 2019

Dedicated to the 100th anniversary of the birth of Professor Ruyu Chen.

Project supported by the National Natural Science Foundation of China (Nos. 21877047, 21867011) and the Project of Science Fund of Jiangxi Education Office (No. GJJ170672).

国家自然科学基金(Nos. 21877047, 21867011)、江西省教育科技厅基金(No. GJJ170672)资助项目. 


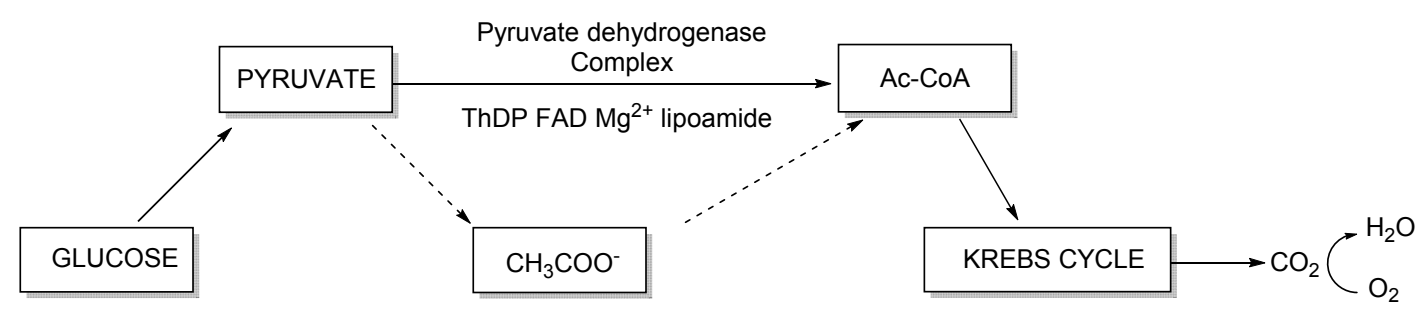

图 1 丙酮酸脱氢酶复合体的催化作用机制

Figure 1 Catalytic mechanism of pyruvate dehydrogenase complex

$\mathrm{E} 1, \mathrm{EC}$ 1.2.4.1), 二氢硫辛酸转乙酰基酶 E2 (dihydrolipoyl transacetylase, PDHc-E2, EC 2.3.1.12)和二氢硫 辛酸脱氢酶 E3 (dihydrolipoyl dehydrogenase, PDHc-E3, EC 1.8.1.4). 如果 PDHc 活性受到抑制, 将导致产生的乙 酰辅酶 $\mathrm{A}$ 减少, 使三羧酸循环不能正常进行, 从而干扰 生物体的正常代谢，最终导致生物体死亡 ${ }^{[7]}$, 其中丙酮 酸脱羧酶(PDHc-E1)是 PDHc 催化反应的起始酶, 其催 化的第一步反应是唯一的不可逆反应. 前期的理论研究 表明, 如果针对 PDHc-E1 设计抑制剂, 干扰第一步关键 反应的正常进行, 将高效导致有害生物体的死亡 ${ }^{[8]}$. 杀 菌剂克菌丹为目前唯一报道的对 PDHc 有抑制作用的广 谱杀菌剂, 但无研究表明克菌丹是针对 PDHc-E1 的抑 制剂 ${ }^{[9]}$. 由于温血动物中的 $\mathrm{PDHc}$ 与微生物中的 $\mathrm{PDHc}$ 的组成结构及同源性存在显著的差异性, 为设计合成高 选择性 PDHc 抑制剂提供了可行性. 因此, 针对国内外 未见 PDHc E1 抑制剂类杀菌剂的研究空白, 开展以 PDHc E1 为靶标的杀菌剂的合理设计, 探索发现具有高 效高选择的 PDHc E1 抑制剂或高效杀菌活性化合物, 具 有很好的研究价值和应用价值.

在 PDHc-E1 催化的过程中需要辅酶 ThDP 的参与才 能进行, 因此目前获得 PDHc-E1 抑制剂的研究策略之 一是通过设计辅酶 ThDP 的类似物来占据 ThDP 的活性 空腔, 以达到抑制 PDHc-E1 活性的目的 ${ }^{[10,11]}$. 在 20 世纪 80 年代国外就已有通过对辅酶 ThDP 的结构修饰来获得 PDHc-E1 抑制剂的研究报道 ${ }^{[12 ~ 15]}$, 但无 PDHc-E1 抑制 剂作为杀菌剂研究应用的报道. 对 ThDP 的结构修饰大 部分是集中在噻唑环，保留焦磷酸结构部分. 该类抑制 剂虽然对 PDHc-E1 具有很好的抑制活性, 但存在结构 复杂、不易合成、选择性差以及焦磷酸结构部分的多电 荷使细胞吸收和生物利用度差等诸多的问题，导致实用 性差 ${ }^{[16]} .2012$ 年, 本课题组贺军波 ${ }^{[17]}$ 首次通过三步合成 获得了对大肠杆菌 (E. coli) PDHc-E1 具有抑制活性的 2甲基-4-氨基-5-(取代- $1 H$-1,2,3-三唑基)甲基嘧啶衍生物 $\mathbf{L}$, 但其杀菌活性却较差(图 2). 本课题组长期致力于丙 酮酸脱氢酶抑制剂的研究, 为本研究奠定了实验基 础 ${ }^{[18 \sim 24]}$.

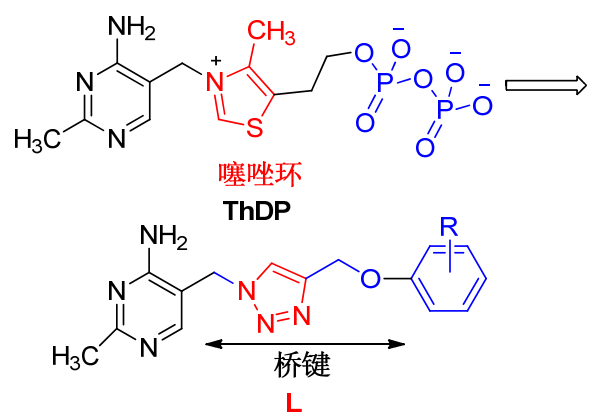

图 2 辅酶 ThDP 的结构修饰

Figure 2 Structural modification of coenzyme ThDP

腙类化合物是由肼与醛或酮缩合而成的一类 Schiff 碱类化合物，许多此类化合物表现出良好的生物活性、 较强的配位能力和多样的配位方式, 因此在医药、农药 等方面具有广泛的应用 ${ }^{[25]}$. 例如许多腙类农药已实现 商品化, 如杀菌剂醌肜腙(Benquiox)、嘧菌腙(Ferimzone)、玉米田苗后除草剂氟吡草腙(Diflufenzopyr)、肠 内杀菌剂硝呋齐特(Nifuroxazide)、化合物 $\mathbf{A}$ 对立枯丝核 菌具有很好的抑制活性等 ${ }^{[26]}$. 此类化合物共同的特点 是含有 $\mathrm{NHN}=$ 活性亚结构单元(图 3)
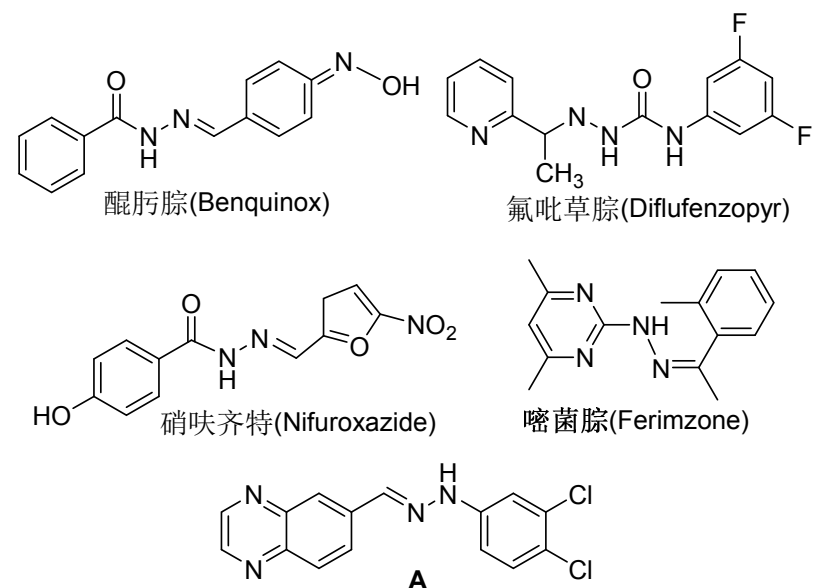

Rhizoctonia solani $\mathrm{EC}_{50}=0.16 \mathrm{mg} / \mathrm{L}$

图 3 含腙结构单元药物

Figure 3 Drugs containing structural units of hydrazone

分析了先导结构 L 在大肠杆菌 PDHc-E1 活性空腔 中的作用模式(图 4), 分子模拟分析发现先导结构中的 
醚键(图 4 红色方框所示)与周围氨基酸残基并无相互作 用，三唑环结构单元与活性空腔中的氨基酸残基 His640 和 Glu522 有较弱的相互作用, 基于 $\mathrm{N}$ 的电负性比 $\mathrm{O}$ 的 电负性弱, 因此 $\mathrm{N}$ 比 $\mathrm{O}$ 对核外电子的束缚能力差, 这样 有利于 $\mathrm{N}$ 核外电子与周围的氨基酸残基形成氢键, 因此 本文利用含孤对电子对的活性亚结构单元一一腙取代 先导结构 $\mathbf{L}$ 中的桥键设计出了目标化合物 $\mathbf{I}$. 期望获得 以 E. coli PDHc-E1 为靶标具有杀菌活性的化合物, 同时 考察桥键的结构变化对其 E. coli PDHc-E1 的抑制活性, 以及杀菌活性的影响. 其设计思路如 Scheme 1 所示. 合 成路线见 Scheme 2.

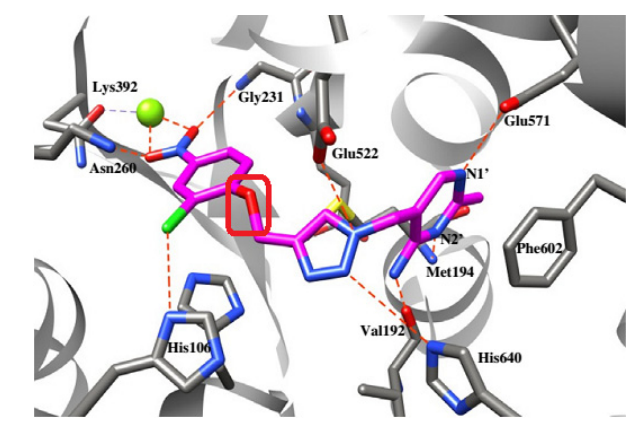

图 4 抑制剂在大肠杆菌 PDHc-E1 中的结合模式 Figure 4 Optimal binding model for inhibitor into the active site of E. coli PDHc-E1<smiles>[R]c1ccccc1NN=Cc1cnc(C)nc1C=Cc1ccc(OCc2cn(CCc3cnc(C)nc3N)nn2)cc1</smiles>

图式 1 目标化合物的设计

Scheme 1 Design of target compounds

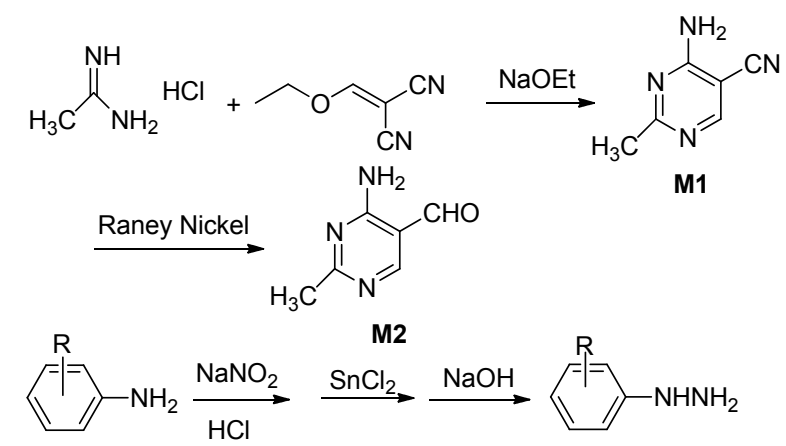

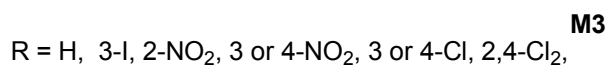

2- $\mathrm{Br}, 3$ or $4-\mathrm{Br}, 2,4-\mathrm{F}_{2}, 2,4,6-\mathrm{Cl}_{3}, 4-\mathrm{CF}_{3}$<smiles>[R]c1cccc(NN=Cc2cnc(C)nc2N)c1</smiles>

图式 2 目标化合物的合成路线

Scheme 2 Synthetic route of the target compound

\section{1 结果与讨论}

\section{1 化合物的合成}

1.1.1中间体 2-甲基-4-氨基-5-甲醛嘧啶(M2)的合成

目标化合物 I 的合成路线中，化合物 M2 是最重要 的中间体. 为了高收率获得中间体 M2, 在合成过程中 需要注意以下几点: (1)制备乙醇钠时，应该在冰浴下加 入钠, 以免温度过高造成体系变黄, 影响下一步收率; (2)加入乙氧基亚甲基丙二腈时，应该用机械搅拌，并且 保证有足够的乙醇, 因为反应开始后体系中有大量的固 体析出产生凝固, 难以搅拌; (3)还原过程中使用的雷尼 镍应该回收循环利用，母液应该用水合肼进行中和后才 能柱层析, 否则产物难以分离. 中间体 M2 的氢谱显示, 嘧啶环上的氨基氢不是单峰, 而是裂分为两重峰，原因 是其中一个氢与羰基上氧成了氢键, 如 Eq. 1 所示.<smiles>Cc1ncc(C=O)c(NCO)n1</smiles>

\subsection{2 目标化合物 I 的合成}

目标化合物 $\mathbf{I}$ 未见文献报道. 根据含腙类似物合成 的相关文献报道, 腙主要是肼与醛在加热条件下脱去一 分子水而生成. 利用此原理来合成目标化合物 I 过程中, 发现肼与醛的投料比对反应有很大的影响. 本文合成目 标化合物 I 所用到的醛为 2-甲基-4-氨基-5-甲醛嘧啶 (M2), 其在乙醇中的溶解性较好, 而肼在乙醇中溶解性 较差. 为了使肼反应完全, 本文采用醛与肼的投料比为 $1.1 ： 1$ 来合成 $\mathbf{I}$, 反应冷却后有白色固体直接析出, 未 反应的醛溶解在乙醇中, 无杂质析出. 反应完成后的体 系可直接加水使目标化合物析出并抽滤，并且杂质可通 过简单的抽滤而除去, 得到的目标化合物再用适量的乙 醇洗去未反应的醛或其它杂质, 即可得到较纯的目标化 合物 I. 此方法操作简单, 无需复杂的后处理程序.

\section{2 目标化合物 I 对 E. coli PDHc-E1 酶抑制活性}

为了篮选发现具有高效杀菌活性的 PDHc-E1 抑制 剂，本文将重点考察所合成的目标化合物 I 对 E. coli PDHc-E1 的酶抑制活性及其杀菌活性，总结目标化合物 I 的结构与活性之间的关系，同时也可探讨目标化合物 的设计思想的合理性.

\subsubsection{E. coli PDHc-E1 酶抑制活性测试方法}

通过本课题组对测活方法及酶动力学条件的系统 研究, 表明采用 2,6-二氯吲哚酚(DCIP)法 ${ }^{[17,18]}$ 具有便于 操作、实验数据相对稳定、且试剂价格便宜等优点, 因 此选择 DCIP 法来测定化合物 I 对 E. coli PDHc-E1 
(CX-ace)活性的影响.

实验原理: 丙酮酸在 PDHc-E1 的催化作用下生成 的羟乙基-ThDP 能够还原 2,6-DCIP，而 2,6-DCIP 在 600 $\mathrm{nm}$ 处有光的吸收, 因此可通过测定 $600 \mathrm{~nm}$ 处吸光度值 的减少量来反映 2,6-DCIP 被还原的量, 进而表示酶活. 吸光度值减少的量越少说明化合物对 PDHc-E1 的抑制 活性越好.

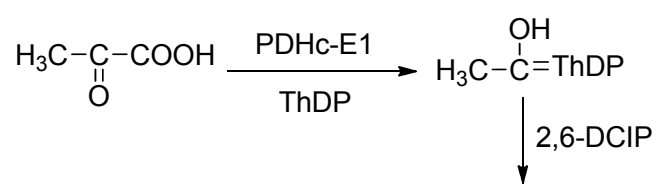

$600 \mathrm{~nm}$ 处吸光度的减少, 反映2,6-DCIP被还原的量

通过上述酶活测试原理来检测化合物对酶活的抑 制作用, 由此来篮选高效的 PDHc-E1 抑制剂, 初篮待测 化合物的终浓度设定为 $100 \mu \mathrm{mol} / \mathrm{L}$. 具体笁选方法如 下.

置 $95 \mu \mathrm{L}$ 反应混合液: $0.05 \mu \mathrm{g} / \mu \mathrm{L}$ PDHc-E1，50 $\mathrm{mmol} / \mathrm{L} \mathrm{K}_{3} \mathrm{PO}_{4}(\mathrm{pH}=6.4), 50 \mu \mathrm{mol} / \mathrm{L}$ ThDP, $0.4 \mathrm{mmol} / \mathrm{L}$ DCIP, $100 \mu \mathrm{mol} / \mathrm{L}$ 用 DMSO 溶解的待测化合物(对照组 加等量的 DMSO), 其余用水补齐, 共配置 4 倍量的反应 液于 $2 \mathrm{~mL}$ 的 $\mathrm{EP}$ 管中, 放置在 $37{ }^{\circ} \mathrm{C}$ 水浴锅中温育 3 $\min$.

酶促反应: 分别取 $95 \mu \mathrm{L}$ 温育后的反应液加入到 96 孔酶标板的 3 个孔中, 迅速加入 $5 \mu \mathrm{L} 1 \mathrm{mmol} / \mathrm{L}$ 的底物 丙酮酸钠开启反应，置于酶标仪中测 $600 \mathrm{~nm}$ 处的吸光 度值, 反应 $0 \mathrm{~min}$ 时测定 $\mathrm{OD}_{0 \mathrm{~min}}, 37{ }^{\circ} \mathrm{C}$ 酶标仪中反应 5 $\min$ 后, 测定 $\mathrm{OD}_{5 \mathrm{~min}}$. 然后求 $5 \mathrm{~min}$ 内的吸光度值改变量 $\Delta \mathrm{OD}_{600}=\mathrm{OD}_{0 \text { min }}-\mathrm{OD}_{5 \mathrm{~min}}$. 每次测定均做溶剂 $\mathrm{DMSO}$ 的对照实验, 每组实验做 3 5 个平行.

(3)数据处理: 化合物引起的吸光度值的改变需扣 除溶剂 DMSO 的影响, $100 \mu \mathrm{mol} / \mathrm{L}$ 待测化合物的抑制率 的计算公式如下:

抑制率 $(\%)=[(\Delta \mathrm{OD}$ 对照 $-\Delta \mathrm{OD}$ 实验 $) / \Delta \mathrm{OD}$ 对照 $] \times$ $100 \%$

1.2.2 目标化合物 I 对 E. coli PDHc-E1 酶抑制活性 如表 1 所示, 部分化合物在 $100 \mu \mathrm{mol} / \mathrm{L}$ 浓度下对 E. coli PDHc-E1 酶显示一定的抑制活性，如化合物 I-1、

I-3、I-4、I-6、I-9、I-11 的抑制率均大于 $60 \%$ ，选择部 分化合物进一步测试了它们的抑制作用 $\mathrm{IC}_{50}$. 如表 2 所 示，除 I-1 的抑制活性优于相应的先导化合物 L-1 外，其 它化合物对 E. coli PDHc-E1 酶的抑制活性较相应的先 导化合物 $\mathbf{L}$ 有所下降, 如化合物 I-3、I-4、I-5. 可能是 结构修饰后 I 的分子链长过短, 造成苯环结构单元难以 到达对应的活性位点, 虽然修饰后的目标化合物对 $E$.
表 1 目标化合物 I 对 E. coli PDHc-E1 的抑制率(\%)

Table 1 Structure and inhibitory activity (\%) of title compounds

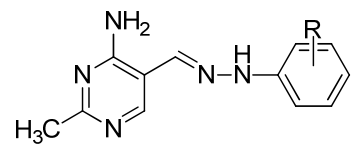

\begin{tabular}{|c|c|c|c|c|c|}
\hline 化合物 & $\mathrm{R}$ & 抑制率/\% & 化合物 & $\mathrm{R}$ & 抑制率 ${ }^{a} / \%$ \\
\hline I-1 & $\mathrm{H}$ & 62.60 & I-8 & $2,4,6-\mathrm{Cl}_{3}$ & 37.10 \\
\hline I-2 & $3-\mathrm{I}$ & 31.56 & I-9 & $4-\mathrm{CF}_{3}$ & 84.52 \\
\hline I-3 & $4-\mathrm{NO}_{2}$ & 68.25 & I-10 & $3-\mathrm{Cl}$ & 34.00 \\
\hline I-4 & $4-\mathrm{Cl}$ & 66.67 & I-11 & $3-\mathrm{Br}$ & 76.89 \\
\hline I-5 & $2,4-\mathrm{Cl}_{2}$ & 0 & I-12 & $2-\mathrm{Br}$ & 39.78 \\
\hline I-6 & $4-\mathrm{Br}$ & 70.93 & I-13 & $3-\mathrm{NO}_{2}$ & 31.44 \\
\hline I-7 & $2,4-\mathrm{F}_{2}$ & 23.81 & I-14 & $2-\mathrm{NO}_{2}$ & 16.44 \\
\hline
\end{tabular}

coli PDHc-E1 的抑制活性较对应的部分先导化合物 $\mathbf{L}$ 有 所降低(表 2), 但此类化合物仍然是以 E. coli PDHc-E1 为靶标的抑制剂, 为后续发现高效 PDHc-E1 酶抑制剂 奠定了实验基础.

表 2 目标化合物 I 对 E. coli PDHc-E1 的 $\mathrm{IC}_{50}$ Table $2 \mathrm{IC}_{50}$ of title compounds $\mathbf{I}$ against $E$. coli $\mathrm{PDHc}-\mathrm{E} 1$<smiles>[R]c1ccc(OCc2cn(Cc3cnc(C)nc3N)nn2)cc1</smiles><smiles>Cc1ncc(/C=N/Nc2ccccc2N)c(N)n1</smiles>

$\mathbf{L}$ I

\begin{tabular}{clc||ccc}
\hline 化合物 & \multicolumn{1}{c|}{$\mathrm{R}$} & $\mathrm{IC}_{50} /\left(\mu \mathrm{mol} \cdot \mathrm{L}^{-1}\right)$ & 化合物 & $\mathrm{R}$ & $\mathrm{IC}_{50} /\left(\mu \mathrm{mol} \cdot \mathrm{L}^{-1}\right)$ \\
\hline $\mathbf{I}-1$ & $\mathrm{H}$ & 29.33 & $\mathbf{I}-5$ & $2,4-\mathrm{Cl}_{2}$ & $>100$ \\
$\mathbf{L}-1$ & $\mathrm{H}$ & 55.15 & $\mathbf{L}-5$ & $2,4-\mathrm{Cl}_{2}$ & 18.74 \\
$\mathbf{I}-3$ & $4-\mathrm{NO}_{2}$ & 30.46 & $\mathbf{I}-6$ & $4-\mathrm{Br}_{1}$ & 26.45 \\
$\mathbf{L}-3$ & $4-\mathrm{NO}_{2}$ & 8.80 & $\mathbf{I}-9$ & $4-\mathrm{CF}_{3}$ & 19.22 \\
$\mathbf{I}-4$ & $4-\mathrm{Cl}$ & 32.65 & $\mathbf{I}-11$ & $3-\mathrm{Br}$ & 22.56 \\
$\mathbf{L}-4$ & $4-\mathrm{Cl}$ & 26.44 & & & \\
\hline
\end{tabular}

\section{3 目标化合物的分子模拟研究}

为了进一步从分子水平理解化合物 $\mathbf{I}$ 与 $E$. coli PDHc-E1 作用靶点的结合模式. 本文借助计算机辅助药 物设计软件 Syby17.3 进行了分子对接模拟研究. 在此研 究中是选取 ThDP/PDHc-E1 复合物的晶体结构 (PDB:1L8A $)^{[27]}$ 作为研究的分子对接模型, E. coli PDHcE1 的辅因子 ThDP 分子占据的位点为分子对接活性腔. 为了理解抑制剂与靶标 PDHc-E1 相互作用模式, 本文 选择对 E. coli PDHc-E1 抑制活性最高的化合物 I-9 进行 了分子对接研究. 其分子对接的结果如图 5 所示.

如图 5 所示, I-9 在 PDHc-E1 活性腔处的结合方式 与本课题组前期研究表明的先导化合物 $\mathbf{L}$ 或 ThDP 在 PDHc-E1 活性腔处的结合方式相似，以 “V” 型构象结 合，可分为三部分进行分析. 在 “ $\mathrm{V}$ ” 型构象的右半部分, I-9 嘧啶环上的氨基与 Val192 形成一个氢键, 氨基嘧啶 


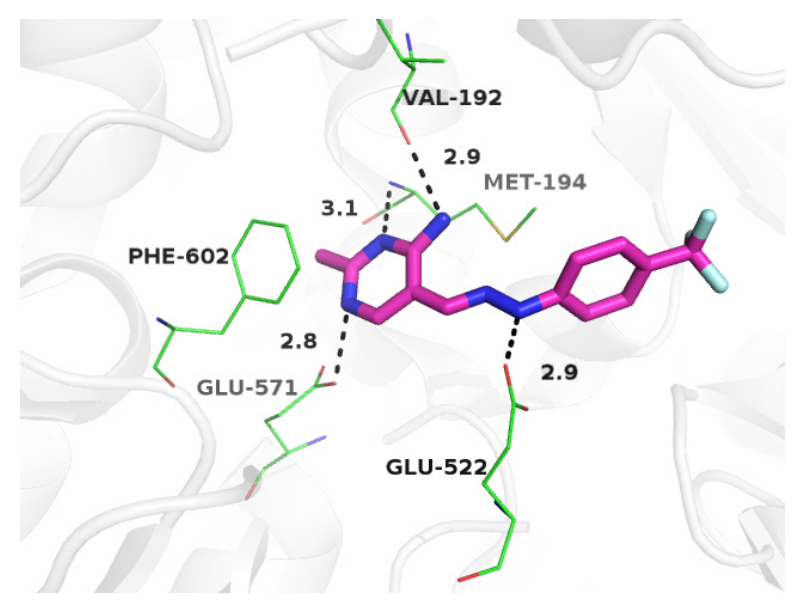

图 5 抑制剂 I-9 在大肠杆菌 PDHc-E1 中的结合模式

Figure 5 Optimal binding model for inhibitor I-9 into the active site of E. coli PDHc-E1

环上的两个氮分别与 Met194 和 Glu571 形成两个氢键. “ $\mathrm{V}$ ” 型构象的中间部分, 腙结构单元上的氮可以与 Glu522 形成氢键作用. 以上氢键作用与先导结构一样, 但是先导结构中的苯环上的取代基与氨基酸残基发生 了氢键作用, 而本文化合物中的苯环上取代基与周围氨 基酸残基无氢键作用, 有可能是碳链过短造成苯环部分 不能到达活性位点, 这也很好地解释了本文设计的化合 物中苯环上有取代基的抑制活性较先导差, 而苯环上无 取代基的抑制活性较先导高. 如 I-3 $\left(\mathrm{R}=4-\mathrm{NO}_{2}\right), \mathbf{I}-4$ $(\mathrm{R}=4-\mathrm{Cl})$ 和 I-5 $\left(\mathrm{R}=2,4-\mathrm{Cl}_{2}\right)$ 对 E. coli PDHc-E1 的抑制 活性均比对应的先导化合物 L-3、L-4、L-5 低, 而苯环 上无取代的 I-1 $(\mathrm{R}=\mathrm{H})$ 对 E. coli PDHc-E1 的抑制活性比 对应的先导化合物 $\mathbf{L - 1}$ 高. 为进一步设计高效 E. coli PDHc-E1 抑制剂指明了方向.

\section{4 目标化合物的杀菌活性}

选择 E. coli PDHc-E1 为微生物的模式靶酶, 其研究 目的是试图通过设计 E. coli PDHc-E1 的高效抑制剂, 并 从中篎选发现对农业病害具有杀菌活性的化合物. 上述 研究结果表明所设计的部分化合物 I 对微生物大肠杆菌 PDHc-E1 具有不同程度的抑制作用, 但是否具有杀菌活 性则成为本文关注的问题. 因此, 本文选择了农业上常 见的 9 种病害为测试靶标, 如代表性真菌: 黄瓜枯萎病 菌(Fusarium oxysporum.sp.cucumebrium Owen)、花生褐 斑病菌(Cercospora arachidicola Hori)、苹果轮纹病菌 (Physalospora piricola Nose)、小麦赤霉病菌(Gibberella zeae)、油菜菌核病菌(Sclerotiniasclerotiorum (Lib.) deBary)、黄瓜灰霉病菌(Botrytis cinerea)、水稻纹枯病菌 (Rhizoctonia solani). 测试浓度为 $50 \mu \mathrm{g} / \mathrm{mL}$, 用十字交叉 法测量菌落直径, 每个培养基测量三次, 取其平均值为 菌落的平均直径, 并通过以下公式计算抑制率, 测试结 果如表 3 所示[对照药剂为百菌清].
菌落扩散直径 $=$ 菌落平均直径一菌饼直径

抑制率 $(\%)=($ 对照菌落扩散直径一处理菌落扩散 直径) $/$ (对照菌落扩散直径) $\times 100 \%$

表 3 目标化合物 I 对真菌的抑制活性(\%)

Table 3 Inhibition rate (\%) of title compounds I against fungus

\begin{tabular}{|c|c|c|c|c|c|c|c|}
\hline 化合物 & $\begin{array}{l}\text { 黄瓜枯 } \\
\text { 萎病菌 }\end{array}$ & 斑病菌 & 纹病 & 㑂 & 核病菌 & 霉病囯 & 枯病茼 \\
\hline I-1 & 50.0 & 54.5 & 73.1 & 38.1 & 70.8 & 50.0 & 50.0 \\
\hline I-2 & 38.9 & 18.2 & 42.3 & 71.4 & 77.1 & 50.0 & 87.5 \\
\hline I-3 & 16.7 & 18.2 & 26.9 & 19.0 & 29.2 & 28.6 & 78.1 \\
\hline I-4 & 61.1 & 63.6 & 73.1 & 76.2 & 83.3 & 64.3 & 84.4 \\
\hline I-5 & 22.2 & 36.4 & 42.3 & 14.3 & 35.4 & 42.9 & 59.4 \\
\hline I-6 & 66.7 & 100.0 & 100.0 & 76.2 & 91.7 & 85.7 & 81.3 \\
\hline I-7 & 38.9 & 100.0 & 100.0 & 66.7 & 68.8 & 85.7 & 68.8 \\
\hline I-8 & 27.8 & 36.4 & 42.3 & 71.4 & 35.4 & 42.9 & 56.3 \\
\hline I-9 & 66.7 & 63.6 & 84.6 & 81.0 & 68.8 & 75.0 & 90.6 \\
\hline I-10 & 50.0 & 72.7 & 76.9 & 81.0 & 70.8 & 60.7 & 84.4 \\
\hline I-11 & 54.5 & 53.3 & 62.5 & 62.5 & 48.3 & 78.8 & 74.1 \\
\hline I-12 & 22.2 & 36.4 & 38.5 & 47.6 & 33.3 & 42.9 & 65.6 \\
\hline I-13 & 27.8 & 27.3 & 34.6 & 33.3 & 14.6 & 7.1 & 40.6 \\
\hline I-14 & 11.1 & 18.2 & 19.2 & 28.6 & 35.4 & 7.1 & 12.5 \\
\hline 百菌清 & 83.3 & 75.0 & 92.3 & 73.1 & 96.4 & 96.1 & 96.1 \\
\hline
\end{tabular}

通过表 3 可以看出，在 $50 \mu \mathrm{g} / \mathrm{mL}$ 浓度下，部分 I 系 列化合物对花生褐斑、苹果轮纹、小麦赤霉、油菜菌核 与水稻纹枯表现出中等至较好的杀菌活性, 其中化合物 I-6 $(\mathrm{R}=4-\mathrm{Br})$ 对小麦赤霉和油菜菌核病菌; I-9 $(\mathrm{R}=$ 4- $\left.\mathrm{CF}_{3}\right)$ 对小麦赤霉和水稻纹枯; $\mathbf{I - 1 0}$ 对小麦赤霉的抑制 活性与商品化杀菌剂百菌清相当. 而本课题组前期文献 报道的先导化合物 $\mathbf{L}$ 对上述菌种显示较弱的抑制活性, 大部分化合物在 $100 \mu \mathrm{g} / \mathrm{mL}$ 浓度下的抑制率小于 $60 \%{ }^{[17]}$.

特别是化合物 I-6 与 I-7 对花生褐斑与苹果轮纹抑 制活性为 $100 \%$, 优于百菌清的抑制活性，具有进一步 研究的价值. 因此我们选择化合物 I-6 与 I-7 进一步测试 了对花生褐斑与苹果轮纹的 $\mathrm{EC}_{50}$ 值, 结果总结如表 4 和 表 5 所示.

表 4 I-6 和 I-7 对花生褐斑病菌的 $\mathrm{EC}_{50}{ }^{a}$

Table $4 \mathrm{EC}_{50}$ of I-6 and I-7 against Cercospora arachidicola Hori

\begin{tabular}{lccc}
\hline 化合物 & $y=a+b x$ & $\mathrm{EC}_{50} /\left(\mu \mathrm{g} \bullet \mathrm{mL}^{-1}\right)$ & $R$ \\
\hline I-6 & $y=1.1356 x+3.6945$ & 14.1125 & 0.9736 \\
I-7 & $y=0.8014 x+4.1105$ & 12.8793 & 0.9937 \\
百菌清 & $y=1.3220 x+3.5711$ & 12.0476 & 0.9565 \\
\hline$a$ 苗活性数据南开大学生测室担供 & &
\end{tabular}

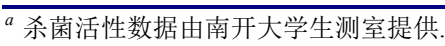


表 5 I-6 和 I-7 对苹果轮纹病菌的 $\mathrm{EC}_{50}{ }^{a}$

Table 5 EC $_{50}$ of I-6 and I-7 against Physalospora piricola Nose

\begin{tabular}{lccc}
\hline 化合物 & $y=a+b x$ & $\mathrm{EC}_{50} /\left(\mu \mathrm{g} \cdot \mathrm{mL}^{-1}\right)$ & $R$ \\
\hline I-6 & $y=0.5755 x+5.1106$ & 0.6425 & 0.9884 \\
I-7 & $y=0.7254 x+5.0720$ & 0.7956 & 0.9836 \\
百菌清 & $y=1.3890 x+3.7981$ & 7.3342 & 0.9862 \\
\hline$a$ 菌
\end{tabular}

a 杀菌活性数据由南开大学生测室提供.

通过对 I-6 和 I-7 以及对照药剂百菌清对花生褐斑 病的毒力曲线测定, 结果发现, 化合物 I-6 和 I-7 对花生 褐斑病菌的 $\mathrm{EC}_{50}$ 值分别为 14.11 和 $12.88 \mu \mathrm{g} / \mathrm{mL}$, 化合物 I-7 对花生褐斑病菌的防治效果较 I-6 好, 并与对照药剂 百菌清活性相当 $\left(\mathrm{EC}_{50}\right.$ 值为 $\left.12.05 \mu \mathrm{g} / \mathrm{mL}\right)$.

通过对 I-6 和 I-7 以及对照药剂百菌清对花生褐斑 病的毒力曲线测定, 结果发现, 化合物 I-6 和 I-7 对苹果 轮纹病的 $\mathrm{EC}_{50}$ 值分别为 0.64 和 $0.80 \mu \mathrm{g} / \mathrm{mL}$, 化合物 I-6 对花生褐斑病的防治效果较 I-7 好, 两者都显著优于百 菌清. 其活性是对照药剂百菌清活性 $\left(\mathrm{EC}_{50}\right.$ 值为 7.33 $\mu \mathrm{g} / \mathrm{mL}$ )的 9 倍以上.

综上结果, I 系列的化合物不仅对 E. coli PDHc-E1 具有一定的抑制活性, 而且其杀真菌活性显著优于先导 化合物 $\mathbf{L}$, 因此, 该结构 $\mathbf{I}$ 具有进一步优化的研究价值, 也表明本文通过设计以微生物中的 PDHc-E1 为靶标的 抑制剂, 可以获得具有杀菌活性的化合物, 本文进一步 测试了这类化合物的除草及杀虫活性. 测试结果表明此 类化合物完全无除草及杀虫活性, 化合物 I 对虫草菌中 的菌具有特定的选择性抑制活性, 符合本文以大肠杆菌 PDHc-E1 为靶标设计杀菌活性化合物的预期设想.

\section{2 结论}

以课题组前期发现的微生物 PDHc-E1 抑制剂 $\mathbf{L}$ 为 先导化合物, 根据 $\mathbf{L}$ 在活性空腔中的作用模式, 在其嘧 啶环与苯环之间引入腙结构单元替代三唑结构单元, 设 计并合成了 14 个新型氨基嘧啶衍生物 I. 目标化合物均 通过 ${ }^{1} \mathrm{H} N M R 、{ }^{13} \mathrm{C} N \mathrm{NMR}$ 、质谱和元素分析进行了结构 表征. 丰富了丙酮酸脱氢酶抑制剂的结构物类型.

生物活性研究发现 I 系列的化合物不仅对 E. coli PDHc-E1 具有一定的抑制活性, 而且杀真菌活性显著优 于先导化合物, 如 I-6 $(R=4-B r)$ 与 $\mathbf{I - 7}\left(\mathrm{R}=2,4-\mathrm{F}_{2}\right)$ 对花 生褐斑的 $\mathrm{EC}_{50}$ 值分别为 $14.11,12.88 \mu \mathrm{g} / \mathrm{mL}$, 与商品化 杀菌剂百菌清 $(12.05 \mu \mathrm{g} / \mathrm{mL})$ 相近或相当, 对苹果轮纹的 $\mathrm{EC}_{50}$ 值分别为 0.64 和 $0.80 \mu \mathrm{g} / \mathrm{mL}$, 是商品化杀菌剂百菌 清抑制效果 $(7.33 \mu \mathrm{g} / \mathrm{mL})$ 的 9 倍以上. 特别是 I-6 不仅对 E. coli PDHc-E1 显示较好的抑制活性 $\left(\mathrm{IC}_{50}=26.45\right.$ $\mu \mathrm{mol} / \mathrm{L})$, 同时对真菌花生褐斑和苹果轮纹显示高效活 性, 具有进一步研究的价值. 以上结果表明通过修饰先 导结构 $\mathbf{L}$ 中的桥键可以获得不仅对 E. coli PDHc-E1 靶
酶具有抑制作用，而且对农业中的有害真菌靶标也具有 显著抑制活性(显著优于先导化合物)的化合物，达到了 结构优化的目的.

\section{3 实验部分}

\section{1 仪器与试剂}

${ }^{1} \mathrm{H}$ NMR 和 ${ }^{13} \mathrm{C}$ NMR 谱用 Varian VNMR $400 \mathrm{MHz}$ 核磁共振仪(氞代二甲亚砜为溶剂); 质谱用 QTRAP 液 质联用仪或 Finnigan Trace Ms 质谱仪; 元素分析用 Vario ELIII CHNSO 型元素分析仪; 熔点采用上海精密 科学仪器有限公司的 WRS-IB 型熔点仪测定(温度未经 校正). 德国 Heidolph 公司生产的 MR3001 型恒温磁力 搅拌器, Buchi R-200 型旋转蒸发仪, Sartotuis 电子天平, ZF-200 暗箱式紫外分析仪, DLSB 低温冷却循环洜, 70-1 型远红外干燥箱等仪器.

盐酸乙脒、雷尼镍、取代苯胺、无水乙醇、氯化亚 锡均为分析纯.

\section{2 实验方法}

3.2.1中间体 2-甲基-4-氨基-5-甲醛嘧啶(M2)的合成 参照文献方法 ${ }^{[28]}$. 在 $100 \mathrm{~mL}$ 单口瓶中加入 $50 \mathrm{~mL}$ 乙醇, 冰盐浴下加入钠 $(4.6 \mathrm{~g}, 0.2 \mathrm{~mol})$ 、盐酸乙脒 $(18.8 \mathrm{~g}$, $0.2 \mathrm{~mol}) 、 乙$ 氧基亚甲基丙二腈 $(24.4 \mathrm{~g}, 0.2 \mathrm{~mol})$, 反应 0.5 $\mathrm{h}$ 过滤得 2-甲基-4-氨基-5-甲腈嘧啶 M1.

在 $250 \mathrm{~mL}$ 三口瓶中加入 M1 (20.1 g, $0.15 \mathrm{~mol})$, 冰 乙酸 $75 \mathrm{~mL}$, 雷尼镍 $5 \mathrm{~g}$, 加热反应, 薄层色谱(TLC)跟 踪反应直至反应完全, 过滤除去雷尼镍, 减压蒸馏除去 乙酸, 柱层析(洗脱剂为: 石油醚/乙酸乙酯, $V: V=1$ : 1)以 75\%收率得到黄色固体 M2. m.p. 197 198 ${ }^{\circ} \mathrm{C} ;{ }^{1} \mathrm{H}$ NMR (400 MHz, DMSO- $\left.d_{6}\right) \delta: 2.41\left(\mathrm{~s}, 3 \mathrm{H}, \mathrm{CH}_{3}\right), 7.91(\mathrm{~s}$, 1H, NH), 8.17 (s, 1H, NH), 8.65 (s, 1H, py-H), 9.82 (s, 1H, $\mathrm{CHOH})$.

\subsection{2中间体取代苯肼(M3)的合成}

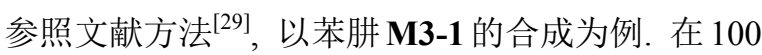
$\mathrm{mL}$ 三口瓶中加入 $30 \%$ 的盐酸 $3 \mathrm{~mL}$ 、水 $2 \mathrm{~mL}$ 、对硝基 苯胺 $(1.38 \mathrm{~g}, 0.01 \mathrm{~mol})$, 降温至 $0 \sim 5{ }^{\circ} \mathrm{C}$, 从液面下的导 管滴加亚硝酸钠 $(0.70 \mathrm{~g}, 0.0101 \mathrm{~mol})$ 和 $2 \mathrm{~mL}$ 水配成的溶 液. 加完搅拌 $30 \mathrm{~min}$ 得橙黄色透明重氮液. 在 $8{ }^{\circ} \mathrm{C}$ 以 下, 将氯化亚锡的盐酸溶液滴加到上述重氮液中, 继续 反应 $2 \mathrm{~h}$. 将反应混合物进行抽滤, 得到淡粉色固体对 硝基苯肼盐酸盐. 在 $100 \mathrm{~mL}$ 的烧杯中加入上述对硝基 苯肼盐酸盐, 适量水, 搅拌 $30 \mathrm{~min}$, 滴加 $30 \%$ 的氢氧化 钠溶液至 $\mathrm{pH}=7$ 得苯肼 M3-1. 直接用于下一步. 用同 样的方法可以制备 M3 其余的中间体. 


\subsection{3 目标化合物 I 的合成通法}

$25 \mathrm{~mL}$ 的单颈圆底烧瓶中分别加入中间体 $\mathbf{M} 2(0.29$ $\mathrm{g}, 2.2 \mathrm{mmol})$ 和各种取代苯肼 $\mathbf{M 3}(2 \mathrm{mmol})$ 并溶于 $10 \mathrm{~mL}$ 无水乙醇. 油浴加热至回流 $3 \mathrm{~h}$, 有白色固体析出, TLC 检测反应完全, 将反应液倒入冷水中, 搅拌有固体析出. 抽滤, 滤饼用少量二氯甲烷洗, 干燥即得目标化合物 $\mathbf{I}$.

2-甲基-4-氨基-5-(甲基苯腙)-嘧啶(I-1)：淡黄色固 体, 产率 85\%, m.p. 249 251 ${ }^{\circ} \mathrm{C} ;{ }^{1} \mathrm{H}$ NMR $(400 \mathrm{MHz}$, DMSO-d $\left.d_{6}\right) \delta: 2.37$ (s, 3H, $\left.\mathrm{CH}_{3}\right), 6.75$ (s, 1H, ArH), 6.91 (d, $J=7.1 \mathrm{~Hz}, 2 \mathrm{H}, \mathrm{ArH}), 7.22(\mathrm{~s}, 2 \mathrm{H}, \mathrm{ArH}), 7.75\left(\mathrm{~s}, 2 \mathrm{H}, \mathrm{NH}_{2}\right)$, $7.90(\mathrm{~s}, 1 \mathrm{H}$, pyrimidine-H), $8.16(\mathrm{~s}, 1 \mathrm{H}, \mathrm{CH}=\mathrm{N}), 11.22(\mathrm{~s}$, $1 \mathrm{H}, \mathrm{NH}) ;{ }^{13} \mathrm{C} \mathrm{NMR}\left(100 \mathrm{MHz}, \mathrm{DMSO}-d_{6}\right) \delta: 164.95$, $163.16,159.19,155.65,144.85,136.37,129.35,119.04$, 108.45, 25.42; ESI-MS $m / z: 228.2[\mathrm{M}+1]^{+}$. Anal. calcd for $\mathrm{C}_{12} \mathrm{H}_{13} \mathrm{~N}_{5}$ : C 63.42, H 5.77, N 30.82; found $\mathrm{C} 63.35, \mathrm{H}$ 5.69 , N 30.59 .

2-甲基-4-氨基-5-(甲基-3-碘苯腙)-嘧啶(I-2): 淡黄 色固体, 产率 79\%. m.p. 242 243 ${ }^{\circ} \mathrm{C} ;{ }^{1} \mathrm{H}$ NMR (400 MHz, DMSO- $\left.d_{6}\right) \delta: 2.37\left(\mathrm{~s}, 3 \mathrm{H}, \mathrm{CH}_{3}\right), 6.95 \sim 7.10(\mathrm{~m}, 3 \mathrm{H}$, ArH), 7.25 (d, $J=1.8 \mathrm{~Hz}, 1 \mathrm{H}, \mathrm{ArH}), 7.73$ (s, 2H, $\mathrm{NH}_{2}$ ), $7.95(\mathrm{~s}, 1 \mathrm{H}$, pyrimidine-H), $8.20(\mathrm{~s}, 1 \mathrm{H}, \mathrm{CH}=\mathrm{N}), 10.59$ (s, $1 \mathrm{H}, \mathrm{NH}) ;{ }^{13} \mathrm{C}$ NMR $\left(100 \mathrm{MHz}, \mathrm{DMSO}-d_{6}\right) \delta: 165.33$, $159.19,156.20,146.28,137.77,131.33,127.27,119.87$, 111.19, 108.15, 95.58, 25.50; ESI-MS $m / z: 354.1[\mathrm{M}+1]^{+}$. Anal. calcd for $\mathrm{C}_{12} \mathrm{H}_{12} \mathrm{IN}_{5}$ : C 40.81, H 3.42, N 19.83; found C 40.81, H 3.52, N 19.91 .

2-甲基-4-氨基-5-(甲基-4-硝基苯腙)-嘧啶(I-3): 红 色固体, 产率 76\%. m.p $>260{ }^{\circ} \mathrm{C} ;{ }^{1} \mathrm{H}$ NMR (400 MHz, DMSO-d $\left.d_{6}\right) \delta: 2.37\left(\mathrm{~s}, 3 \mathrm{H}, \mathrm{CH}_{3}\right), 7.01(\mathrm{~d}, J=8.4 \mathrm{~Hz}, 2 \mathrm{H}$, ArH), 7.70 (s, 2H, $\left.\mathrm{NH}_{2}\right), 8.03$ (s, 1H, pyrimidine-H), 8.09 (d, $J=8.8 \mathrm{~Hz}, 2 \mathrm{H}, \mathrm{ArH}), 8.23(\mathrm{~s}, 1 \mathrm{H}, \mathrm{CH}=\mathrm{N}), 11.22$ (s, $1 \mathrm{H}, \mathrm{NH}) ;{ }^{13} \mathrm{C}$ NMR $\left(100 \mathrm{MHz}, \mathrm{DMSO}-d_{6}\right) \delta: 166.24$, $159.31,157.50,149.94,141.71,138.45,126.29,111.05$, 107.48, 25.55; ESI-MS m/z: $273.4[\mathrm{M}+1]^{+}$. Anal. calcd for $\mathrm{C}_{12} \mathrm{H}_{12} \mathrm{~N}_{6} \mathrm{O}_{2}$ : C 52.94, $\mathrm{H} 4.44, \mathrm{~N} 30.87$; found $\mathrm{C} 52.63$, $\mathrm{H} 4.31, \mathrm{~N} 30.71$.

2-甲基-4-氨基-5-(甲基-4-氯苯腙)-嘧啶(I-4)：黄色 固体, 产率 71\%. m.p. 240 241 ${ }^{\circ} \mathrm{C} ;{ }^{1} \mathrm{H}$ NMR $(400 \mathrm{MHz}$, DMSO-d $\left.d_{6}\right) \delta: 2.37\left(\mathrm{~s}, 3 \mathrm{H}, \mathrm{CH}_{3}\right), 6.93(\mathrm{~d}, J=8.7 \mathrm{~Hz}, 2 \mathrm{H}$, Ar-H), 7.27 (d, J=8.6 Hz, 2H, ArH), 7.73 (s, 2H, $\mathrm{NH}_{2}$ ), $7.92(\mathrm{~s}, 1 \mathrm{H}$, pyrimidine-H), $8.20(\mathrm{~s}, 1 \mathrm{H}, \mathrm{CH}=\mathrm{N}), 10.49(\mathrm{~s}$, $1 \mathrm{H}, \mathrm{NH}) ;{ }^{13} \mathrm{C}$ NMR $\left(100 \mathrm{MHz}, \mathrm{DMSO}-d_{6}\right) \delta: 165.27$, $159.20,156.13,143.79,137.36,129.14,122.24,113.22$, 108.24, 25.49; ESI-MS m/z: $262.3[\mathrm{M}+1]^{+}$. Anal. Calcd for $\mathrm{C}_{12} \mathrm{H}_{12} \mathrm{ClN}_{5}$ : C 55.07, H 4.62, N 26.76; found C 55.00,
H 4.41, N 26.37 .

2-甲基-4-氨基-5-(甲基-2,4-二氯苯腙)-嘧啶(I-5)：淡 黄色固体，产率 69\%. m.p. 239 241 ${ }^{\circ} \mathrm{C} ;{ }^{1} \mathrm{H}$ NMR (400 $\left.\mathrm{MHz}, \mathrm{DMSO}-d_{6}\right) \delta: 2.38\left(\mathrm{~s}, 3 \mathrm{H}, \mathrm{CH}_{3}\right), 7.26(\mathrm{~s}, 1 \mathrm{H}, \mathrm{ArH})$, 7.31 (s, 1H, Ar-H), 7.47 (s, 1H, ArH), 7.73 (s, 2H, $\mathrm{NH}_{2}$ ), $8.17(\mathrm{~s}, 1 \mathrm{H}$, pyrimidine-H), $8.37(\mathrm{~s}, 1 \mathrm{H}, \mathrm{CH}=\mathrm{N}), 10.03(\mathrm{~s}$, $1 \mathrm{H}, \mathrm{NH}) ;{ }^{13} \mathrm{C}$ NMR (100 MHz, DMSO- $\left.d_{6}\right) \delta: 165.86$, $159.34,156.81,141.39,140.15,128.83,128.22,122.44$, 117.02, 114.28, 107.89, 25.53; ESI-MS m/z: $296.3[\mathrm{M}+$ $1]^{+}$. Anal. calcd for $\mathrm{C}_{12} \mathrm{H}_{11} \mathrm{Cl}_{2} \mathrm{~N}_{5}: \mathrm{C} 48.67, \mathrm{H} 3.74, \mathrm{~N}$ 23.65; found $\mathrm{C} 48.75, \mathrm{H} 3.85, \mathrm{~N} 23.61$.

2-甲基-4-氨基-5-(甲基-4-溴苯腙)-嘧啶(I-6)：黄色 固体, 产率 77\%. m.p. 237 238 ${ }^{\circ} \mathrm{C} ;{ }^{1} \mathrm{H}$ NMR (400 MHz, DMSO-d $\left.d_{6}\right) \delta: 2.37\left(\mathrm{~s}, 3 \mathrm{H}, \mathrm{CH}_{3}\right), 6.89$ (d, $J=7.8 \mathrm{~Hz}, 2 \mathrm{H}$, Ar-H), 7.38 (d, J=7.8 Hz, 2H, ArH), 7.75 (s, 2H, NH$)_{2}$, $7.93(\mathrm{~s}, 1 \mathrm{H}$, pyrimidine-H), $8.20(\mathrm{~s}, 1 \mathrm{H}, \mathrm{CH}=\mathrm{N}), 10.51(\mathrm{~s}$, $1 \mathrm{H}, \mathrm{NH}) ;{ }^{13} \mathrm{C}$ NMR (100 MHz, DMSO- $\left.d_{6}\right) \delta: 165.14$, $163.29,159.27,155.80,144.14,137.35,131.96,113.75$, 108.29, 25.38; ESI-MS m/z: $306.2[\mathrm{M}+1]^{+}$. Anal. Calcd for $\mathrm{C}_{12} \mathrm{H}_{12} \mathrm{BrN}_{5}$ : C 47.08, H 3.95, N 22.87; found C 47.00, $\mathrm{H} 4.15, \mathrm{~N} 22.55$.

2-甲基-4-氨基-5-(甲基-2,4-二氟苯腙)-嘧啶(I-7): 黄色固体, 产率 71\%. m.p. 222 224 ${ }^{\circ} \mathrm{C} ;{ }^{1} \mathrm{H}$ NMR (400 MHz, DMSO-d $d_{6} \delta: 2.37$ (s, 3H, $\left.\mathrm{CH}_{3}\right), 7.02(\mathrm{~s}, 1 \mathrm{H}, \mathrm{ArH})$, $7.22(\mathrm{~d}, 2 \mathrm{H}, \mathrm{ArH}, J=8.7 \mathrm{~Hz}), 7.74\left(\mathrm{~s}, 2 \mathrm{H}, \mathrm{NH}_{2}\right), 8.15$ (s, $1 \mathrm{H}$, pyrimidine-H), $8.15(\mathrm{~s}, 1 \mathrm{H}, \mathrm{CH}=\mathrm{N}), 10.20(\mathrm{~s}, 1 \mathrm{H}$, $\mathrm{NH}) ;{ }^{13} \mathrm{C}$ NMR (100 MHz, DMSO- $\left.d_{6}\right) \delta: 165.40,163.15$, $159.28,156.15,139.45,130.07,113.73,111.67,111.47$, 108.12, 104.40, 104.16, 103.90, 25.43; ESI-MS m/z: 264.1 $[\mathrm{M}+1]^{+}$. Anal. calcd for $\mathrm{C}_{12} \mathrm{H}_{11} \mathrm{~F}_{2} \mathrm{~N}_{5}$ : C 54.75, H 4.21, N 26.60; found $\mathrm{C} 54.41, \mathrm{H} 4.39, \mathrm{~N} 26.84$.

2-甲基-4-氨基-5-(甲基-2,4,6-三氯苯腙)-嘧啶(I-8): 淡黄色固体，产率 79\%. m.p $>260{ }^{\circ} \mathrm{C} ;{ }^{1} \mathrm{H}$ NMR (400 $\left.\mathrm{MHz}, \mathrm{DMSO}-d_{6}\right) \delta: 2.35$ (s, 3H, $\left.\mathrm{CH}_{3}\right), 7.64$ (s, 2H, Ar-H), $7.72\left(\mathrm{~s}, 2 \mathrm{H}, \mathrm{NH}_{2}\right), 8.13$ (s, 1H, pyrimidine-H), $8.20(\mathrm{~s}, 1 \mathrm{H}$, $\mathrm{CH}=\mathrm{N}), 9.69(\mathrm{~s}, 1 \mathrm{H}, \mathrm{NH})$; ESI-MS $m / z: 330.1[\mathrm{M}+1]^{+}$. Anal. calcd for $\mathrm{C}_{12} \mathrm{H}_{10} \mathrm{Cl}_{3} \mathrm{~N}_{5}$ : C 43.60, $\mathrm{H}$ 3.05, N 21.18; found $\mathrm{C} 43.73, \mathrm{H} 2.96, \mathrm{~N} 21.31$.

2-甲基-4-氨基-5-(甲基-4-三氟甲基苯腙)-嘧啶(I-9): 淡黄色固体，产率 $84 \%$. m.p. 252 254 ${ }^{\circ} \mathrm{C} ;{ }^{1} \mathrm{H} \mathrm{NMR}$ $\left(400 \mathrm{MHz}, \mathrm{DMSO}-d_{6}\right) \delta: 2.38\left(\mathrm{~s}, 3 \mathrm{H}, \mathrm{CH}_{3}\right), 7.06$ (d, $J=8.4$ $\mathrm{Hz}, 2 \mathrm{H}, \mathrm{ArH}), 7.56$ (d, J=8.5 Hz, 2H, ArH,), 7.75 (s, 2H, $\left.\mathrm{NH}_{2}\right), 8.00(\mathrm{~s}, 1 \mathrm{H}$, pyrimidine-H), $8.24(\mathrm{~s}, 1 \mathrm{H}, \mathrm{CH}=\mathrm{N})$, $10.83(\mathrm{~s}, 1 \mathrm{H}, \mathrm{NH}) ;{ }^{13} \mathrm{C} \mathrm{NMR}\left(100 \mathrm{MHz}, \mathrm{DMSO}-d_{6}\right) \delta$ : $165.67,159.31,156.67,147.77,138.99,126.68,123.72$, 
119.17, 118.86, 118.54, 118.22, 111.48, 107.99, 25.50; ESI-MS 295.9 $[\mathrm{M}+1]^{+}$Anal. Calcd for $\mathrm{C}_{13} \mathrm{H}_{12} \mathrm{~F}_{3} \mathrm{~N}_{5}$ : C 52.88, H 4.10, N 23.72; found C 52.63, H 4.32, N 23.53.

2-甲基-4-氨基-5-(甲基-3-氯苯腙)-嘧啶(I-10): 淡黄 色固体, 产率 80\%. m.p. 246 248 ${ }^{\circ} \mathrm{C} ;{ }^{1} \mathrm{H}$ NMR (400 $\left.\mathrm{MHz}, \mathrm{DMSO}-d_{6}\right) \delta: 2.37\left(\mathrm{~s}, 3 \mathrm{H}, \mathrm{CH}_{3}\right), 6.78(\mathrm{~d}, J=7.7 \mathrm{~Hz}$, $1 \mathrm{H}, \operatorname{ArH}), 6.87$ (d, $J=8.3 \mathrm{~Hz}, 1 \mathrm{H}, \operatorname{ArH}), 7.24$ (t, $J=8.0$ $\mathrm{Hz}, 1 \mathrm{H}, \mathrm{ArH}), 7.74$ (s, 2H, $\mathrm{NH}_{2}$ ), 7.94 (s, 1H, pyrimidine-H), $8.22(\mathrm{~s}, 1 \mathrm{H}, \mathrm{CH}=\mathrm{N}), 10.56(\mathrm{~s}, 1 \mathrm{H}, \mathrm{NH})$; ESI-MS $m / z: 262.3[\mathrm{M}+1]^{+}$. Anal. calcd for $\mathrm{C}_{12} \mathrm{H}_{12} \mathrm{ClN}_{5}$ : C 55.07, H 4.62, N 26.76; found C 55.04, H 4.44, N 26.54.

2-甲基-4-氨基-5-(甲基-3-溴苯腙)-嘧啶(I-11)：黄色 固体，产率 $79 \%$. m.p $>260{ }^{\circ} \mathrm{C} ;{ }^{1} \mathrm{H}$ NMR $(400 \mathrm{MHz}$, DMSO- $\left.d_{6}\right) \delta: 2.37\left(\mathrm{~s}, 3 \mathrm{H}, \mathrm{CH}_{3}\right), 6.92(\mathrm{dd}, J=8.0,2.0 \mathrm{~Hz}$, 2H, ArH), 7.06 (t, $J=2.0 \mathrm{~Hz}, 2 \mathrm{H}, \mathrm{ArH}), 7.19$ (t, $J=8.0 \mathrm{~Hz}$, 1H, ArH), 7.74 (s, 2H, $\left.\mathrm{NH}_{2}\right), 7.94$ (s, 1H, pyrimidine-H), $8.22(\mathrm{~s}, 1 \mathrm{H}, \mathrm{CH}=\mathrm{N}), 10.55(\mathrm{~s}, 1 \mathrm{H}, \mathrm{NH})$; ESI-MS $m / z$ : $306.2[\mathrm{M}+1]^{+}$. Anal. calcd for $\mathrm{C}_{12} \mathrm{H}_{12} \mathrm{BrN}_{5}$ : C 47.08, $\mathrm{H}$ 3.95, N 22.87; found C 47.30, H 4.00, N 22.69.

2-甲基-4-氨基-5-(甲基-2-溴苯腙)-嘧啶(I-12): 绿色 固体, 产率 $82 \%$. m.p $>260{ }^{\circ} \mathrm{C} ;{ }^{1} \mathrm{H} \mathrm{NMR}(400 \mathrm{MHz}$, DMSO- $\left.d_{6}\right) \delta: 2.38\left(\mathrm{~s}, 3 \mathrm{H}, \mathrm{CH}_{3}\right), 6.77(\mathrm{t}, J=7.5 \mathrm{~Hz}, 1 \mathrm{H}$, $\operatorname{ArH}), 7.23$ (d, $J=8.0 \mathrm{~Hz}, 1 \mathrm{H}, \operatorname{ArH}), 7.33$ (t, $J=7.7 \mathrm{~Hz}$, 1H, ArH), 7.52 (d, J=7.9 Hz, 1H, ArH), 7.77 (s, 2H, $\mathrm{NH}_{2}$ ), $8.17(\mathrm{~s}, 1 \mathrm{H}$, pyrimidine-H), $8.38(\mathrm{~s}, 1 \mathrm{H}, \mathrm{CH}=\mathrm{N}), 9.66(\mathrm{~s}$, $1 \mathrm{H}, \mathrm{NH})$; ESI-MS $m / z: 306.0[\mathrm{M}+1]^{+}$. Anal. Calcd for $\mathrm{C}_{12} \mathrm{H}_{12} \mathrm{BrN}_{5}$ : C 47.08, H 3.95, N 22.87; found $\mathrm{C} 47.03, \mathrm{H}$ 3.88, N 22.97.

2-甲基-4-氨基-5-(甲基-3-硝基苯腙)-嘧啶(I-13)：淡 黄色固体，产率 84\%. m.p. 255 257 ${ }^{\circ} \mathrm{C} ;{ }^{1} \mathrm{H}$ NMR (400 MHz, DMSO- $\left.d_{6}\right) \delta: 2.38\left(\mathrm{~s}, 3 \mathrm{H}, \mathrm{CH}_{3}\right), 7.35(\mathrm{~d}, J=7.9 \mathrm{~Hz}$, $1 \mathrm{H}, \mathrm{ArH}), 7.50$ (t, 1H, Ar-H, $J=8.0 \mathrm{~Hz}), 7.58(\mathrm{~d}, 1 \mathrm{H}$, Ar-H, $J=8.0 \mathrm{~Hz}$ ), 7.68 (s, 1H, Ar-H), 7.76 (s, 2H, NH$)_{2}$, $8.00(\mathrm{~s}, 1 \mathrm{H}$, pyrimidine- $\mathrm{H}), 8.26(\mathrm{~s}, 1 \mathrm{H}, \mathrm{CH}=\mathrm{N}), 10.89(\mathrm{~s}$, $1 \mathrm{H}, \mathrm{NH})$; ESI-MS m/z: $273.1[\mathrm{M}+1]^{+}$. Anal. calcd for $\mathrm{C}_{12} \mathrm{H}_{12} \mathrm{~N}_{6} \mathrm{O}_{2}$ : C 52.94, H 4.44, N 30.87; found C 52.57, $\mathrm{H}$ $4.42, \mathrm{~N} 30.53$.

2-甲基-4-氨基-5-(甲基-2-硝基苯腙)-嘧啶(I-14)：淡 黄色固体, 产率 56\%. m.p. 220 222 ${ }^{\circ} \mathrm{C} ;{ }^{1} \mathrm{H}$ NMR (400 $\left.\mathrm{MHz}, \mathrm{DMSO}-d_{6}\right) \delta: 2.57$ (s, $\left.3 \mathrm{H}, \mathrm{CH}_{3}\right), 7.00$ (s, $\left.1 \mathrm{H}, \mathrm{ArH}\right)$, $7.73(\mathrm{dd}, J=6.6,8.0 \mathrm{~Hz}, 2 \mathrm{H}, \mathrm{ArH}), 8.12$ (d, $J=8.1 \mathrm{~Hz}$, $1 \mathrm{H}, \mathrm{ArH}), 8.57$ (d, $\left.J=6.6,15.2 \mathrm{~Hz}, 2 \mathrm{H}, \mathrm{NH}_{2}\right), 8.69$ (s, $1 \mathrm{H}$, pyrimidine-H), $9.68(\mathrm{~s}, 1 \mathrm{H}, \mathrm{CH}=\mathrm{N}), 11.28(\mathrm{~s}, 1 \mathrm{H}, \mathrm{NH})$; ESI-MS $m / z$ : $273.1[\mathrm{M}+1]^{+}$. Anal. calcd for $\mathrm{C}_{12} \mathrm{H}_{12} \mathrm{~N}_{6} \mathrm{O}_{2}$ : C 52.94, H 4.44, N 30.87; found C 52.84, H 4.55, N 30.67.
辅助材料(Supporting Information) 产物的核磁共振 (氢谱、碳谱)谱图。这些材料可以免费从本刊网站 (http://sioc-journal.cn/)上下载.

\section{References}

[1] Bates, D. L.; Danson, M. J.; Hale, G.; Hopper, E. A.; Perham, R. N. Nature 1977, 268, 313.

[2] Perham, R. N. Annu. Rev. Biochem. 2000, 69, 961.

[3] Wei, W.; Li, H.; Nemeria, N.; Jordan, F. Protein Expression Purif. 2003, 28, 140 .

[4] Dobritzsch, D.; König, S.; Schneider, G.; Lu, G. J. Biol. Chem. 1998, 273, 20196.

[5] Kluger, R.; Pike, D. C. J. Am. Soc. Chem. 1977, 99, 4504.

[6] Kern, D.; Kern, G.; Neef, H.; Tittmann, K.; Killenberg-Jabs, M.; Wikner, C.; Schneider, G.; Hübner, G. Science 1997, 275, 67.

[7] Nemeria, N.; Baykal, A.; Joseph, E.; Yan, S. Z.; Furey, W.; Jordan, F. Biochemistry 2004, 43, 6565.

[8] Wang, J. Y.; Dong, H.; Li, S. H.; He, H. W. J. Phys. Chem. B 2005, 109, 18664.

[9] Tang, C. Z. Pesticide Chemistry, Nankai University Press, Tianjing, 2000, The front page, p. 304 (in Chinese) (唐除痴, 农药化学, 南开大学出版社, 天津, 2000, 头版, p. 304.)

[10] Gutowski, J. A.; Lienhard, G. E. J. Biol. Chem. 1976, 251, 2863.

[11] Agyei-Owusu, K.; Leeper, F. J. FEBS J. 2009, 276, 2905.

[12] Lowe, P.; Leeper, F. J.; Perham, R. N. Biochemistry 1983, 22, 150.

[13] Arjunan, P.; Chandrasekhar, K.; Sax, M.; Brunskill, A.; Nemeria, N.; Jordan, F.; Furey, W. Biochemistry 2004, 43, 2405.

[14] Arjunan, P.; Sax, M.; Brunskill, A.; Chandrasekhar, K.; Nemeria, N.; Zhang, S.; Jordan, F.; Furey, W. J. Biol. Chem. 2006, 281, 15296.

[15] Erixon, K. M.; Dabalos, C. L.; Leeper, F. J. Chem. Commun. 2007, 960.

[16] Nemeria, N.; Yan, Y.; Zhang, Z.; Brown, A.M.; Arjunan, P.; Furey,W.; Guest, J. R.; Jordan, F. J. Biol. Chem. 2001, 276, 45969.

[17] He, J. B.; Feng, L. L.; Li, J.; Tao, R. J.; Wang, F.; Liao, X.; Sun, Q. S.; Long, Q. W.; Ren, R. L.; Wan, J.; He, H. W. Bioorg. Med. Chem. 2012, 20, 1665.

[18] He, J. B.; Feng, L. L.; Li, J.; Tao, R. J.; Ren, Y. L.; Wan, J.; He, H. W. Bioorg. Med. Chem. 2014, 22, 89 .

[19] Feng, L. L.; He, J. B.; He, H. F.; Zhao, L. L.; Deng, L. F.; Zhang, L.; Zhang, L.; Ren, Y. L.; Wan, J.; He, H. W. Org. Biomol. Chem. 2014, 12, 8911 .

[20] He, J. B.; He, H. F.; Zhao, L. L.; Zhang, L.; You, G. Y.; Feng, L. L.; Wan, J.; He, H. W. Bioorg. Med. Chem. 2015, 23, 1395.

[21] He, H. F.; Feng, J.T.; He, J. B.; Xia, Q.; Ren, Y. L.; Wang, F.; Peng, H.; He, H. W.; Feng, L. L. RSC Adv. 2016, 6, 4310.

[22] He, H. F.; Wang, W.; Zhou, Y.; Xia, Q.; Ren, Y. L.; Feng, J. T.; Peng, H.; He, H. W.; Feng, L. L. Bioorg. Med. Chem. 2016, 24, 1879.

[23] Zhou, Y.; Zhang, S. S.; He, H. W.; Jiang, W.; Hou, L. F.; Xie, D.; Cai, M.; Peng, H.; Feng, L. L. Bioorg. Med. Chem. 2018, 26, 84.

[24] He, H. F.; Xia, H. Y.; Xia, Q.; Ren, Y. L.; He, H. W. Bioorg. Med. Chem. 2017, 25, 5652

[25] Anand, P.; Patil, V.; Sharma, V.; Khosa, R.; Masand, N. Int. J. Drug Delivery 2012, 3, 851 .

[26] Kang, S. H.; Hu, D. Y.; Wu, J.; Song, B. A. Pesticide 2012, 51, 238 (in Chinese). (康圣鸿，胡德禹，吴剑，宋宝安，农药, 2012, 51, 238.)

[27] Arjunan, P.; Nemeria, N.; Brunskill, A.; Chandrasekhar, K.; Sax, M.; Yan, Y.; Jordan, F.; Guest, J. R.; Furey, W. Biochemistry 2002, 41, 5213.

[28] Anderson, K.; Chen, Y.; Chen, Z.; Luk, K. C.; Rossman, P. L.; Sun, H. M.; Wovkulich, P. M. US 2012/184542, 2012.

[29] Fu, C. Fine Spec. Chem. 2004, 12, 14 (in Chinese). (付春, 精细与专用化学品, 2004, 12, 14.) 